Results Compared with Wistar-Kyoto rats (WKY), whole body and myocardial insulin sensitivity decreased in SHR as manifested by increased fasting blood glucose (6.24 \pm 0.21 vs.5.18 $\pm 0.19 \mathrm{mmol} / \mathrm{l}, \mathrm{n}=6, \mathrm{p}<0.05)$ and decreased insulin-induced cardiac function changes especially for \pm LVdp/dtmax respectively, which was partly attributable to decreased PPAR $\gamma$ expression in myocardium $(0.72 \pm 0.08$ vs $1.08 \pm 0.07, n=4, p<0.05)$. Moreover, 9-week swimming training not only attenuated the fasting blood glucose $(5.54+0.16$ vs $6.24 \pm 0.21 \mathrm{mmol} / \mathrm{l}, \mathrm{n}=6$, $\mathrm{p}<0.05)$ improved cardiac function and enhanced myocardial response to insulin in vivo in SHR, but also increased myocardial PPAR $\gamma$ and subsequent Akt expressions (1.18 \pm 0.12 vs 0.72 $\pm 0.08, \mathrm{n}=4, \mathrm{p}<0.01$ and $0.953 \pm 0.13$ vs $0.514 \pm 0.14, \mathrm{n}=4$, $\mathrm{p}<0.05)$ in SHR.

Conclusions These data demonstrate that 9-week swimming training increased myocardial PPAR $\gamma$ and subsequent Akt expressions in SHR, which is partly involved in improved myocardial insulin sensitivity. The present findings also indicate that the decreased PPAR $\gamma$ expression and subsequent phosphatidylinositol 3-kinase (PI-3 kinase)/Akt signalling perhaps plays a causative role in the impaired inotropic response to insulin in SHR heart Thus, AE merges as an important choice in future SHR preclinical and clinic investigation .

\section{GW23-e2071 LONG-TERM AEROBIC EXERCISE INCREASES MYOCARDIAL PPAR $\gamma$ EXPRESSION IN SPONTANEOUSLY HYPERTENSIVE RATS}

doi:10.1136/heartjnl-2012-302920a.115

Kun-Ru Zhang, Wei Wang, Su-Li Guo, Ai-Jing Qu, Kun-Ru Zhang. Sports College of Shaanxi Normal University

Objectives It is well known that cardiac insulin resistance exists in spontaneously hypertensive rats (SHR), which is attributable to decreased peroxisome proliferator-activated receptor-gamma (PPAR $\gamma$ ) expression in myocardium. However, the effects of aerobic exercise (AE) on myocardial insulin sensitivity in SHR rats are largely unclear. Therefore, the present study aimed to determine the effects of 9-week swimming training on myocardial insulin sensitivity in SHR and the underlying mechanism, with the special focus on the role of exercise in myocardial PPAR $\gamma$ expression.

Methods 4-weeks-old SHR were randomly subjected to 9 weeks of either sedentary or freeloading swimming exercise ( $2 \mathrm{~h} /$ day, $5 \mathrm{~d} /$ week). Blood glucose, cardiac systolic/diastolic function and $\operatorname{PPAR} \gamma$, protein kinase B (Akt) expressions in myocardium were determined at the end of exercise. 